\title{
Angiogenic Switch
}

National Cancer Institute

\section{Source}

National Cancer Institute. Angiogenic Switch. NCI Thesaurus. Code C19664.

Loss of the balance between stimulation and inhibition of new blood vessel growth. A required early step in cancer growth and metastasis. 